This postprint is published in:

Matematičeskie zametki, ISSN 0025-567X, Volume 109, Issue 3, 2021, pp. 370-379

DOI: $10.4213 / \mathrm{mzm} 12845$

УДЕ 512.554

\title{
Функции роста кодлины неассоциативных алгебр
}

\section{М. В. Зайцев, Д. Д. Реповш}

Рассматриваются числовые характеристики тождеств неассоциативных алгебр. Построена серия алгебр с субэкспоненциальной функцией роста кодлины.

\section{1. Введение}

Применение асимптотических методов в различных областях алгебры и дискретной математики является общепринятой практикой современных исследований. В качестве примеров можно привести изучение разнообразнных функций роста в теории формальных языков [1], в теории групп [2], а также в теории полиномиальных тождеств [3]. В теории тождественных соотношений линейных алгебр изучение количественных характеристик играет в последние годы все более значительную роль (см., например, работу [4] и библиографию в ней). Важнейшими из таких характеристик являются так называемые последовательности коразмерностей $\left\{c_{n} A\right\}$ и кодлин $\left\{l_{n} A\right\}$ заданной алгебры $A$ (основые определения и понятия мы напомним в следующем разделе).

Первая из упомянутых характеристик исследована гораздо детальнее. Тем не менее, к настоящему моменту появилось достаточно много работ, анализирующих поведение последовательности $\left\{l_{n}(A)\right\}$ для различных алгебр $A$. Одной из первых работ в указанном направлении была пубикация [5], в которой доказано, что последовательность $\left\{l_{n}(A)\right\}$ растет полиномиально для любой ассоциативной алгебры с нетривиальным тождеством, т.е. РІ-алгебры. Важность этого результата обусловлена тем, что многие другие характеристики растут экспоненциально и влиянием роста кодлины можно пренебречь. Заметим также, что если $A$ - свободная ассоциативная алгебра, то рост $\left\{l_{n}(A)\right\}$ сверхэкспоненциален.

Работа первого автора поддержана грантом „Структурная теория и комбинаторно-логические методы в теории алгебраических систем“ Московского центра фундаментальной и прикладной математики МГУ им. М.В.Ломоносова, работа второго автора поддержана грантом Словенского исследовательского агенства P1-0292. 
В случае алгебр Ли последовательность $\left\{l_{n}(A)\right\}$ демонстрирует более сложное поведение. С одной стороны, класс алгебр Ли $L$ с полиномиальным ростом $\left\{l_{n}(L)\right\}$ достаточно широк. В него входят все конечномерные адгебры, алгебры Ли с нильпотентным коммутантом, аффинные алгебры Каца - Муди и ряд других. С другой стороны, известны примеры (см. [6]) алгебр Ли $L$ с $l_{n}(L) \sim(\sqrt{b})^{n}$ для любых целых $b \geqslant 2$, и даже алгебр со сверхэкспоненциальным ростом кодлины. Например, если $L-$ свободная разрешимая ступени три алгебра Ли счетного ранга, то

$$
l_{n}(L) \sim \frac{n !}{(\ln n)^{n}},
$$

(см. [6]). Есть и примеры промежуточного роста, но их немного. Приведем один из них: если $L$ порождает многообразие $\mathbf{A} N_{2}$, то согласно [7]

$$
l_{n}(L) \sim \exp \left(\pi \sqrt{\frac{2 n}{3}}\right) .
$$

В общем неассоциативном случае имеется лишь ряд разрозненных результатов. Так, если $A$ - конечномерная алгебра произвольной сигнатуры, $\operatorname{dim} A=d$, то

$$
l_{n}(A) \leqslant d(n+1)^{d^{2}+d},
$$

как доказано в [8]. В работах [8], [9] построено семейство примеров бесконечномерных двуступенно левонильпотентных алгебр с полиномиальным ростом последовательностей $\left\{l_{n}\right\}$. Приведем еще один любопытный пример. В работе [10] показано, что существует ровно три многообразия $\mathbf{V}=\operatorname{var} A$, для которых $l_{n}(\mathbf{V})=1$ для всех $n=1,2, \ldots$. Одно из них порождено ассоциативно-коммутативной алгеброй многочленов $F[t]$, другое - двумерной метабелевой алгеброй Ли, а третье - бесконечномерной йордановой алгеброй $J$, построенной И.П. Шестаковым [11; стр. 104, пример 2].

Основная цель данной статьи - построение семейства примеров с субэкспоненциальным ростом последовательности $\left\{l_{n}(A)\right\}$ (теорема 1 и ее следствия). При этом характер асимптотического поведения $\left\{l_{n}(A)\right\}$ может быть как монотонным, так и сильно осциллирующим. Примеры базируются на новом подходе к построению неассоциативных алгебр при помощи бесконечных двоичных слов, предложенном в [12], [13] и развитом в [14], [15]. Вновь предложенная конструкция позволяет связать числовые инварианты алгебр с комбинаторными характеристиками бесконечных слов и использовать результаты хорошо развитой теории формальных языков.

\section{2. Основные понятия и определения}

Пусть $F$ - поле нулевой характеристики. Обозначим через $F\{X\}$ абсолютно свободную алгебру над $F$ с бесконечным множеством порождающих $X$. Если $A-$ $F$-алгебра, то полином $f=f\left(x_{1}, \ldots, x_{n}\right) \in F\{X\}$ называется тождеством алгебры $A, f\left(a_{1}, \ldots, a_{n}\right)=0$ для любых $a_{1}, \ldots, a_{n} \in A$. Все необходимые сведения по теории тождественных соотношений можно найти в [3] или в [16]. Совокупность всех тождеств алгебры $A$ образует идеал $I d(A)$ в $F\{X\}$ устойчивый относительно всех эндоморфизмов $F\{X\}$, т.е. Т-идеал. Обозначим через $P_{n}$ подпространство всех полилинейных многочленов в $F\{X\}$ от $x_{1}, \ldots, x_{n}$. Тогда $P_{n} \cap I d(A)$ - это совокупность 
всех полилинейных тождеств алгебрв $A$ степени $n$. Хорошо известно, что в случае поля нулевой характеристики любой Т-идеал однозначно определяется своими полилинейными компонентами. Поэтому изучение тождеств алгебры $A$ во многом сводится к исследованию семейства подпространств $P_{n} \cap \operatorname{Id}(A), n=1,2, \ldots$ Как правило, удобнее рассматривать семейство факторпространств

$$
P_{n}(A)=\frac{P_{n}}{P_{n} \cap I d(A)} .
$$

При исследовании полилинейных тождеств важную роль играет теория представлений симметрической группы $S_{n}$. Действие $S_{n}$ на полилинейных одночленах задается соотношением

$$
\sigma \circ f\left(x_{1}, \ldots, x_{n}\right)=f\left(x_{\sigma(1)}, \ldots, x_{\sigma(n)}\right),
$$

превращающее $P_{n}$ в $F S_{n}$-модуль. Пространство $P_{n} \cap I d(A)$ инвариантно относительно действия $S_{n}$, поэтому структурой $F S_{n}$-модуля наделено и пространство $P_{n}(A)$. Его характер $\chi\left(P_{n}(A)\right)$ называется $n$-м кохарактером $A$ и обозначается как $\chi_{n}(A)$. Необходимые сведения по теории представлений симметрических групп можно найти в монографии [17]. Разложение $P_{n}(A)$ в сумму неприводимых слагаемых удобно записывать в терминах характеров как

$$
\chi_{n}(A)=\sum_{\lambda \vdash n} m_{\lambda} \chi_{\lambda}
$$

где $\chi_{\lambda}$ - неприводимый характер, соответствующий разбиению $\lambda$ числа $n$, а неотрицательное целое $m_{\lambda}-$ кратностью вхождения $\chi_{\lambda}$ в $\chi_{n}(A)$. Необходимо напомнить, что разбиением $\lambda$ числа $n$ называется набор целых чисел $\lambda=\left(\lambda_{1}, \ldots, \lambda_{k}\right)$, такой, что $\lambda_{1} \geqslant \ldots \geqslant \lambda_{k}>0, \lambda_{1}+\cdots+\lambda_{k}=n$. Размерность соответствующего неприводимого представления (или степень характера) обозначается как $d_{\lambda}$ или $\operatorname{deg} \chi_{\lambda}$. Величина

$$
l_{n}(A)=\sum_{\lambda \vdash n} m_{\lambda}
$$

называется $n$-й кодлиной $A$. Другими словами, $l_{n}(A)-$ количество слагаемых в разложении $F S_{n}$-модуля $P_{n}(A)$ в сумму неприводимых компонент.

Нам потребуется еще одна количественная характеристика, связанная с тождествами алгебры $A$. Напомним, что $n$-й коразмерностью тождеств алгебры $A$ называется величина

$$
c_{n}(A)=\operatorname{dim} P_{n}(A)
$$

Очевидно, что

$$
c_{n}(A)=\sum_{\lambda \vdash n} m_{\lambda} \operatorname{deg} \chi_{\lambda},
$$

в котором $m_{\lambda}-$ те же, что и в $(2.1)$.

Поскольку мы рассматриваем неассоциативные алгебры, важную роль играет расстановка скобок на одночленах различных алгебр. Обозначим через $T$ одну из расстановок скобок на слове длины $n$, а через $\left[a_{1} \cdots a_{n}\right]_{T}$ - произведение $n$ элементов неассоциативной алгебры с этой расстановкой скобок. Например, если $n=4, \mathrm{a}$ $T=(\cdot)(\cdot)$, то $\left[x_{1}, \ldots, x_{4}\right]_{T}=\left(x_{1} x_{2}\right)\left(x_{3} x_{4}\right)$. Алгебру $A$ с тождеством

$$
\left(x_{1} x_{2}\right)\left(x_{3} x_{4}\right) \equiv 0
$$


мы будем называть метабелевой.

При фиксированной расстановке скобок $T$ можно рассматривать в $P_{n}$ подпространство $P_{n}^{T}$, порожденное всеми одночленами $\left[x_{\sigma(1)} \cdots x_{\sigma(n)}\right]_{T}, \sigma \in S_{n}$. Ясно, что

$$
P_{n}=\bigoplus_{T} P_{n}^{T},
$$

где сумма берется по всем возможным расстановкам скобок т.е. содержит

$$
\frac{1}{n}\left(\begin{array}{c}
2 n-2 \\
n-1
\end{array}\right)
$$

слагаемых. Каждое из подпространств $P_{n}^{T}$ является $F S_{n}$-подмодулем в $P_{n}$, как и $P_{n} \cap \operatorname{Id}(A)$. Поэтому структурой $F S_{n}$-модуля наделен и фактормодуль

$$
P_{n}^{T}(A)=\frac{P_{n}^{T}}{P_{n}^{T} \cap I d(A)},
$$

характер которого мы будем обозначать как $\chi_{n}^{T}(A)$.

Нам потребуется следующий результат из работы [12]. Обозначим через $M_{1}$ свободную метабелеву алгебру с одним порождающим $z$. Тогда

$$
\chi_{n}^{T}\left(M_{1}\right)=\chi_{n}+2 \chi_{(n-1,1)}
$$

для любой расстановки скобок $T$, для которой $\left[z_{1} \cdots z_{n}\right]_{T} \neq 0$ в $M_{1}$.

\section{3. Основные результаты}

Напомним конструкцию алгебры ассоциированной с бесконечным двоичным словом. Пусть $w=w_{1} w_{2} \ldots$, где все $w_{i}$ равны 0 или 1 . Комбинаторной сложностью слова $w$ называется функция $C o m p_{w}: \mathbb{N} \rightarrow \mathbb{N}$, где $C o m p_{w}(n)$ - число различных подслов в $w$ длины $n$.

Пусть теперь $w$ - бесконечное слово в алфавите $\{0 ; 1\}$. Обозначим через $A(w)$ неассоциативную алгебру с базисом $\left\{a, b_{0}, b_{1}, \ldots\right\}$, умножение в котором задается соотношением

$$
b_{k}=\left\{\begin{array}{lll}
a b_{k-1}, & \text { если } & w_{k}=1 \\
b_{k-1} a, & \text { если } & w_{k}=0
\end{array}\right.
$$

для всех $k \geqslant 1$, а все остальные произведения равны нулю. При любом слове $w$ алгебра $A(w)$ удовлетворяет тождеству $(2.3)$, поэтому все $F S_{n}$-разложения для $P_{n}(A)$ можно рассматривать по модулю соотношения (2.3), т.е. не в самой алгебре $F\{X\}$, а в свободной метабелевой алгебре $M\{X\}$.

Для элемента $x$ любой метабелевой алгебры обозначим через $R_{x}$ и $L_{x}$ операторы правого и левого умножения на $x$ соответственно. И тот и другой операторы удобно писать справа, т.е. $y R_{x}=y x, y L_{x}=x y$. Для любого двоичного слова $u=u_{1} \ldots u_{m}$ и для $y, x_{1}, \ldots, x_{m} \in X \subset M\{X\}$ обозначим через $y u\left(x_{1}, \ldots, x_{m}\right)$ одночлен $y T_{1} \cdots T_{m}$, где $T_{i}=R_{x_{i}}$, если $u_{i}=0$, или $T_{i}=L_{x_{i}}$, если $u_{i}=1, i=1, \ldots, m$. Нетрудно заметить, что любой одночлен степени $n$ в $M\{X\}$ записывается в виде

$$
\left(x_{i} x_{j}\right) u\left(x_{i_{1}}, \ldots, x_{i_{n-2}}\right),
$$


где $u$ - двоичное слово длины $n-2$.

Пусть $w=w_{1} w_{2} \ldots$ - бесконечное двоичное слово. Конечное слово $u$ мы будем называть собственным подсловом $w$, если $u$ - подслово слова $w_{2} w_{3} \cdots$.

Лемма 1. Полилинейный одночлен $\left(y_{1} y_{2}\right) u\left(x_{1}, \ldots, x_{m}\right)$ не является тождеством алгебры $A(w)$ тогда и только тогда, когда и-собственное подслово $w$.

ДокАЗАтЕЛЬСтво. Пусть $u=w_{i} \ldots w_{i+m-1}$ и $i \geqslant 2$. Тогда $b_{i-2} T_{a} u(a, \ldots, a)=$ $b_{i+m-1} \neq 0$, где $T_{a}=R_{a}$, если $w_{i-1}=0$, либо $T_{a}=L_{a}$, если $w_{i-1}=1$. Следовательно, $\left(y_{1} y_{2}\right) u\left(x_{1}, \ldots, x_{m}\right) \notin I d\left(A(w)\right.$. С другой стороны, $y_{1} y_{2}$ на базисных элементах алгебры $A(w)$ принимает только значения $b_{1}, b_{2}, \ldots$. Но тогда при $k \geqslant 1$ произведение $b_{k} u(a, \ldots, a) \neq 0$ только для $u=w_{k+1} \ldots w_{k+m}$.

Поскольку $A(w)$ не порождается одним элементом, мы не можем непосредственно воспользоваться соотношением (2.6), чтобы ограничить кодлину этой алгебры. Обозначим через $\widetilde{A}(w)$ ее подалгебру, порожденную элементом $a+b_{0}$.

Лемма 2. Алгебры $A(w)$ и $\widetilde{A}(w)$ удовлетворяют одним и тем же тождествам, m.e. $\operatorname{Id}(A(w))=\operatorname{Id}(\widetilde{A}(w))$.

ДокАЗАтЕльство. Заметим сначала, что $\widetilde{A}(w)$ - линейная оболочка элементов $a+b_{0}, b_{1}, b_{2}, \ldots$ Поскольку характеристика поля $F$ равна нулю, достаточно сравнить полилинейные тождества двух алгебр. Включение $\operatorname{Id}(A(w)) \subseteq I d(\widetilde{A}(w))$ очевидно, поскольку $\widetilde{A}(w)$ - подалгебра $A(w)$.

Покажем теперь, что любой полилинейный многочлен $f=f\left(x_{1}, \ldots, x_{n}\right)$ не являющийся тождеством $A(w)$, не равен нулю тождественно и в $\widetilde{A}(w)$. Поскольку обе алгебры метабелевы, можно считать $f$ полиномом свободной метабелевой алгебры $M\{X\}$. В этом случае $f$ можно записать в виде линейной комбинации

$$
f=\sum_{i, k} \sum_{J} \sum_{u} \alpha_{i, k, J, u}\left(x_{i} x_{k}\right) u\left(x_{j_{1}}, \ldots, x_{j_{n-2}}\right),
$$

где $J=\left\{j_{1}, \ldots, j_{n-2}\right\}=\{1, \ldots, n\} \backslash\{1,2\}, u$ - двоичное слово длины $n-2$, в соответствии с (3.1).

Если $f$ не является тождеством $A(w)$, то существует подстановка

$$
\varphi: X \rightarrow\left\{a, b_{0}, b_{1}, \ldots\right\}
$$

такая, что $\varphi(f) \neq 0$.

Ясно, что при этом $\varphi\left(x_{i_{0}}\right)=b_{m}$ ровно для одного из индексов $i_{0}$ и $\varphi\left(x_{r}\right)=a$ для всех остальных $r$. При этом ровно одно из двух произведений $x_{i_{0}} x_{k}, x_{k} x_{i_{0}}$ принимает ненулевое значение $b_{m+1}$. Пусть, например, $b_{m} a=b_{m+1}, a b_{m}=0$. Тогда при подстановке $\varphi$ в ноль переходят все одночлены $\left(x_{i} x_{k}\right) u\left(x_{j_{1}}, \ldots, x_{j_{n-2}}\right)$ с $i \neq i_{0}$. Далее, в ноль переходят все $\left(x_{i_{0}} x_{k}\right) u\left(x_{j_{1}}, \ldots, x_{j_{n-2}}\right)$ с $u \neq u_{0}$, где $u_{0}=w_{m+2} \ldots w_{n+m-1}$ - подслово $w$, начинающееся с $m+2$-й буквы. Запишем $f$ в виде суммы двух слагаемых $f=f_{0}+f_{1}$, где

$$
\begin{gathered}
f_{0}=\sum_{k} \sum_{J} \alpha_{i_{0}, k, J, u_{0}}\left(x_{i_{0}} x_{k}\right) u_{0}\left(x_{j_{1}}, \ldots, x_{j_{n-2}}\right) \\
f_{1}=\sum_{i \neq i_{0}} \sum_{k} \sum_{J} \sum_{u} \alpha_{i, k, J, u}\left(x_{i} x_{k}\right) u\left(x_{j_{1}}, \ldots, x_{j_{n-2}}\right)+
\end{gathered}
$$




$$
+\sum_{k} \sum_{J} \sum_{u \neq u_{0}} \alpha_{i_{0}, k, J, u}\left(x_{i_{0}} x_{k}\right) u\left(x_{j_{1}}, \ldots, x_{j_{n-2}}\right) .
$$

Тогда $\varphi\left(f_{1}\right)=0$, a $\varphi\left(f_{0}\right)=\lambda\left(b_{m} a\right) u_{0}(a, \ldots, a)=\lambda b_{m+n-1}$, где

$$
\lambda=\sum_{k} \sum_{J} \alpha_{i_{0}, k, J, u_{0}} \neq 0 .
$$

Теперь заменим подстановку $\varphi$ на $\widetilde{\varphi}$, такую что $\widetilde{\varphi}\left(x_{i}\right)=a+b_{0}$ для всех $i=1, \ldots, n$. Тогда $\widetilde{\varphi}\left(x_{i_{0}} x_{k}\right)=b_{m} a=b_{m+1}, \widetilde{\varphi}\left(x_{i} x_{j}\right)=0$ для всех $i \neq i_{0}$. В частности, $\widetilde{\varphi}\left(f_{1}\right)=0$ и $\widetilde{\varphi}\left(f_{0}\right)=\varphi\left(f_{0}\right)$, т.е. $\widetilde{\varphi}(f)=\varphi(f) \neq 0$. Поскольку $\widetilde{\varphi}\left(x_{i}\right) \in \widetilde{A}(w)$ для всех $i=1, \ldots, n$, то $f$ не является тождеством $\widetilde{A}(w)$, и лемма доказана.

Перейдем к доказательству основного результата статьи. Разделим собственные подслова в $w$ на две категории. Подслово $u$ назовем подсловом 1-го типа, если оно встречается в $w$ только после нуля, либо только после единицы. Если $u$ встречается в $w$ и после 0 и после 1 , то назовем его подсловом 2-го тuпа.

Tеорема 1. Пусть $w=w_{1} w_{2} \ldots$ - бесконечное двоичное слово. Тогда

$$
l_{n}(A(w))=2 k_{n-2}^{(1)}+3 k_{n-2}^{(2)},
$$

где $k_{m}^{(1)}, k_{m}^{(2)}$ - количество собственных подслов 1-го и 2-го типов длины $m$ в $w$ соответственно. В частности,

$$
2 \operatorname{Comp}_{w^{*}}(n-2) \leqslant l_{n}(A(w)) \leqslant 3 \operatorname{Comp}_{w^{*}}(n-2),
$$

где $w^{*}=w_{2} w_{3} \ldots$

ДоказАтЕльСтво. Сначала уточним строение $P_{n}^{T}(A)$ из $(2.5)$ и разложение пространства $P_{n}(A)$ в сумму таких $P_{n}^{T}(A)$ в случае $A=A(w)$. Заметим, что все полилинейные одночлены вида (3.1) с одним и тем же двоичным словом $u$ имеют одинаковую расстановку скобок и не равны нулю в свободной метабелевой алгебре $M\{X\}$. Кроме того, различным $u$ соответствуют различные расстановки скобок. В частности, пространство $P_{n, u}$ - линейная оболочка полилинейных одночленов (3.1) с фиксированным $u$ в $F\{X\}$ - совпадает с одним из подпространств $P_{n}^{T}$ из (2.4), причем для такой расстановки скобок $T$, что $P_{n}^{T} \nsubseteq I d(M\{X\})$. И наоборот, если $P_{n}^{T} \not I I d(M\{X\})$, то для такой расстановки скобок $T$ найдется слово $u$, при котором $P_{n}^{T}=P_{n, u}$. С учетом леммы 1 , мы приходим к выводу, что

$$
P_{n} \equiv \sum_{u} P_{n, u}(\bmod I d(A(w))),
$$

где сумма берется по всем собственным подсловам $u$ слова $w$.

Пусть $u_{1}, \ldots, u_{N}$ - все собственные подслова $w$ длины $n-2$. Докажем, что

$$
P_{n} \cap \operatorname{Id}(A(w))=P_{n, u_{1}} \cap I d(A(w)) \oplus \cdots \oplus P_{n, u_{N}} \cap I d(A(w))+\sum_{T^{\prime}} P_{n}^{T^{\prime}},
$$

где $T^{\prime}$ пробегает все расстановки скобок, для которых $P_{n}^{T^{\prime}} \subset I d(A(w))$. Очевидно, что правая часть (3.5) лежит в левой. Поэтому достаточно доказать, что если $f_{1}+$ $\cdots+f_{N} \equiv 0$ - тождество алгебры $A(w)$, то и все $f_{1}, \ldots, f_{N^{-}}$тоже тождества $A(w)$. 
Пусть, например, $u_{1}=w_{k+1} \ldots w_{k+n-2}, k \geqslant 1$. Тогда при любой подстановке $\varphi: X \rightarrow\left\{a, b_{0}, b_{1}, \ldots\right\}$, такой, что $\varphi\left(x_{i_{0}}\right)=b_{k-1}$ для некоторого $i_{0}, \varphi\left(x_{t}\right)=a, t \neq i_{0}$, все элементы $(3.1)$ с $u \neq u_{1}$ переходят в ноль поскольку $b_{k} u_{1}(a, \ldots, a)=b_{k+n-1}$, а $b_{k} u(a, \ldots, a)=0$. В частности, $\varphi\left(f_{2}\right)=\ldots=\varphi\left(f_{N}\right)=0$. Следовательно, и $\varphi\left(f_{1}\right)=0$. Если же $\varphi^{\prime}-$ другая подстановка, у которой $\varphi^{\prime}\left(x_{0}\right)=b_{m-1}, m \geqslant 1$, a $u_{1} \neq$ $u_{m+1} \ldots u_{m+n-2}$, то также $\varphi^{\prime}\left(f_{1}\right)=0$. Следовательно, $f_{1} \in I d(A(w))$. Аналогично, $f_{2}, \ldots, f_{N}$ - тождества $A(w)$, и соотношение (3.5) доказано.

Из (3.5) с учетом (2.5) получаем разложение

$$
P_{n}(A(w))=\bigoplus_{u} P_{n, u}(A(w)),
$$

в котором суммирование ведется по всем собственным подсовам $u$ слова $w$ длины $n-2, \mathrm{a}$

$$
P_{n, u}(A(w))=\frac{P_{n, u}}{P_{n, u} \cap I d(A(w))} .
$$

Таким образом, чтобы вычислить длину $F S_{n}$-модуля $P_{n}(A(w))$, т.е. $l_{n}(A(w))$, достаточно подсчитать и просуммировать величины

$$
l_{n, u}(A(w))=\sum_{\lambda \vdash n} m_{\lambda}^{(u)},
$$

где

$$
\chi\left(P_{n, u}(A(w))\right)=\sum_{\lambda \vdash n} m_{\lambda}^{(u)} \chi_{\lambda} .
$$

Согласно лемме 1 и соотношению (2.6), имеем:

$$
\chi\left(P_{n, u}(A(w))\right)=r \chi_{(n)}+s \chi_{n-1,1},
$$

где $r=0$ или $1, s=0,1$ или 2 для любого собственного подслова $u$ в $w$. При этом выполняется равенство

$$
\operatorname{dim} P_{n, u}(A(w))=r+s(n-1)
$$

поскольку $\operatorname{deg} \chi_{(n)}=1, \operatorname{deg} \chi_{n-1,1}=n-1$.

В лемме 4 работы [15] показано, что $\operatorname{dim} P_{n, u}(A(w))=n$, если $u$ - собственное подслово 1-го типа. Легко заметить, чо равенство (3.7) возможно лишь при $r=$ $s=1$, а значит $l_{n, u}(A(w))=2$. Для подслова $u$ второго типа в той же статье (см. лемму 5 и замечание 1 ) доказано, что $\operatorname{dim} P_{n, u}(A(w))=2 n-1$, что влечет равенства $r=1, s=2$ и $l_{n, u}(A(w))=3$. Отсюда следует соотношение (3.3), а из него - равенство (3.4), поскольку $k_{n-2}^{(1)}+k_{n-2}^{(2)}=\operatorname{Comp}_{w^{*}}(n-2)$.

Полученный результат позволяет реализовать широкий класс функций в качестве функций роста кодлины. Приведем несколько примеров субэкспоненциального роста.

СлЕДСТвиЕ 1. Пусть $\varphi: \mathbb{R}^{+} \rightarrow \mathbb{R}^{+}-$такая функиия, что

1) $\varphi(t)>>\log _{2}(t)$;

2) $\varphi$ дифберениируема на $(0 ; \infty)$;

3) $\varphi^{\prime}(t)<<t^{-\beta}$ для некоторой константы $\beta>0$; 
4) $\varphi-$ убъвающал функиия.

Тогда существует алгебра $A$, для которой $l_{n}(A) \sim 2^{\varphi(n)}$.

ДоКАЗАТЕЛЬСтво. В работе [18] доказано, что для любой $\varphi(t)$ с условиями $(1)-$ (4) существует двоичное слово $w$, для которого $\operatorname{Comp}_{w}(n) \sim 2^{\varphi(n)}$. Теперь достаточно применить теорему 1 к алгебре $A(w)$.

В формулировке следствия 1 соотношение $f(t)<<g(t)$ означает, что

$$
\lim _{t \rightarrow \infty} \frac{f(t)}{g(t)}=0 .
$$

Следствие 1 реализует как монотонные субэкспоненциальные функции, например, $2^{n^{\alpha}}$ или $n^{n^{\alpha}}$ c $\alpha \in(0 ; 1)$, так и слабоосцилирующие функции. Более экзотический пример, приведенный в [18], соответствует функции

$$
\varphi(t)=(t+10)^{\frac{1}{2}+\frac{1}{4} \cos (\ln \ln (t+1))},
$$

которая медленно осциллирует между $n^{1 / 2}$ и $n^{3 / 4}$.

Используя другие результаты теории формальных языков мы можем строить примеры алгебр с гораздо более резкими колебаниями последовательности $\left\{l_{n}(A)\right\}$. Так, в работе [19] (теорема 3) предъявлен пример двоичного слова $w$, у которого комбинаторная сложность колеблется от ,почти линейной“ до „почти экспоненциальной“. Используя упомянутый пример, получаем такой результат.

СледСтвиЕ 2. Существует алгебра А вида $A(w)$, для которой можсно выбрать возрастающую последовательность $n_{k}, k=1,2, \ldots$, так, что

(a) $l_{n_{k}}<n_{k}+\ln \ln n_{k}$, если $k$ четно;

(b) $l_{n_{k}}>2^{\frac{n_{k}}{\ln \ln n_{k}}}$, если $k$ нечетно.

Кроме реализации функций с экзотической асимптотикой теорема 1 позволяет в ряде случаев вычислять точные значения кодлины. В теории факториальных языков хорошо известен язык $E_{0}$, который состоит из всех слов в двухбуквенном алфавите $\{a, b\}$, не содержащих в качестве подслов $a^{2}, b^{4}$ и $a b b a$ (см., например, [1]). Несложно построить бинарное слово $\bar{w}$, у которого множество собственных подслов совпадает с языком $E_{0}$. Один из таких примеров приведен в [15]. Там же для слова $\bar{w}$ показано, что

a

$$
k_{n-2}^{(1)}=\left\{\begin{array}{lll}
F_{k-1}+F_{k+1} & \text { при } & n=2 k \\
F_{k-1}+F_{k+2} & \text { при } & n=2 k+1,
\end{array}\right.
$$

$$
k_{n-2}^{(2)}=F_{k}
$$

и при $n=2 k$ и при $n=2 k+1$, где

$$
F_{t}=\frac{\varphi^{t}+(-\varphi)^{-t}}{2 \varphi-1}-\text { числа Фибоначчи, } \varphi=\frac{1+\sqrt{5}}{2} .
$$

Применяя теорему 1 , получаем еще одно следствие.

СлЕДСТвиЕ 3. Для алгебры $A(\bar{w})$ выполняются равенства

$$
l_{n}(A(\bar{w}))=2 F_{t+1}+F_{t+3}
$$


при четном $n=2 t u$

$$
l_{n}(A(\bar{w}))=F_{t+1}+2 F_{t+3}
$$

при нечетном $n=2 t+1$.

Наряду с точным значением можно оценить и асимптотику последовательности $l_{n}(A(\bar{w}))$. Если ввести на вещественных функциях отношение

$$
f(x) \simeq g(x) \Leftrightarrow \lim _{x \rightarrow \infty} \frac{f(x)}{g(x)}=1,
$$

TO

- $l_{n}(A(\bar{w})) \simeq C_{0}(\sqrt{\varphi})^{n}$ при четных $n$

- $l_{n}(A(\bar{w})) \simeq 2 C_{0}(\sqrt{\varphi})^{n}$ при нечетных $n$, где

$$
C_{0}=\frac{\varphi^{2}}{2}=\frac{3+\sqrt{5}}{4}
$$

\section{NIENOE ЦЕOE?OBAННОЙ ЛЕОА?АОУ?U}

[1] А. М. Шур, "О вычислении параметров и типов поведения комбинаторной сложности регулярных языков", Тр. ИММ УрО РАH, 16:2 (2010), 270-287.

[2] Р.И. Григорчук, "Степени роста конечно-порожденных групп и теория инвариантных средних", Изв. АН СССР. Сер. матем., 84:3 (1984), 939-985.

[3] A. Giambruno, M. Zaicev, Polynomial identities and asymptotic methods, American Mathematical Society, Providence, 2005.

[4] М. В. Зайцев, С.П. Мищенко, "Последовательности коразмерностей тождеств и их асимптотическое поведение", Фундаментальная и прикладная математика, 22:4 (2019), 115-127.

[5] A. Berele, A. Regev, "Applications of hook Young diagrams to P.I. algebras", J. Algebra, 82 (1983), 559-567.

[6] S. P. Mishchenko, M. V. Zaicev, "Asymptotic behaviour of colength of varieties of Lie algebras", Serdica Math.J., 26:2 (2000), 45-154.

[7] A. Giambruno, S. Mishchenko, M. Zaicev, "On colength of a variety of Lie algebras", Internat. J. Algebra Comput., 9:5 (1999), 483-491.

[8] A. Giambruno, S. Mishchenko, M. Zaicev, "Algebras with intermediate growth of the codimensions", Adv. Appl. Math., 37:3 (2006), 360-377.

[9] A. Giambruno, S. Mishchenko, M. Zaicev, "Codimensions of Algebras and Growth Functions", Adv. Math., 217:3 (2008), 1027-1052.

[10] С. П. Мищенко, "Многообразия линейных алгебр кодлины один", Вестн. Моск. ун-та. Сер. 1, Математика. Механика, 2010, № 1, 25-30.

[11] К.А. Жевлаков, А. М. Слинько, И. П. Шестаков, А. И. Ширшов, Кольиа, близкие к ассоциативным, Наука, М., 1978.

[12] С. П. Мищенко, А. Б. Веревкин, "О многообразиях с тождествами однопорожденной свободной метабелевой алгебры", Чебышевский сборник, $\mathbf{1 7 : 2}(2016), 21-55$. 
[13] С. П. Мищенко, "Бесконечные периодические слова и почти нильпотентные многообразия", Вестн. Моск. ун-та. Сер. 1, Математика. Механика, 2017, № 4, 62-66.

[14] М. В. Зайцев, Д.Д. Реповш, "Тождества в алгебрах и комбинаторные свойства двоичных слов", Доклады Академии наук, 489:5 (2019), 449-451.

[15] М. В. Зайцев, Д. Д. Реповш, “Теория формальных языков и тождества неассоциативных алгебр", Сиб. мат. журн., 61:2 (2020), 322-329.

[16] Ю.А. Бахтурин, Тождества в алгебрах Ли, Наука, М., 1985.

[17] Г. Джеймс, Теория представлений симметрических групп, Мир, М., 1982.

[18] J. Cassaigne, "Constructing infinite words of intermediate complexity", Lect. Notes Comp. Sci., 2450 (2003), 173-184.

[19] J. Balogh, B. Bollobas, "Hereditary properties of words.", Theor. Inform. Appl., 39:1 (2005), 49-65.

\section{М. В. Зайцев}

Механико-математический факультет, Московский центр фундаментальной и прикладной математики МГУ им. М.В. Ломоносова, г. Москва

E-mail: zaicevmv@mail.ru

\section{Д. Д. Реповш}

Педагогический факультет, Факультет математики и физики университета Любляны, Институт

математики, физики и механики, г. Любляна,

Словения

E-mail: dusan.repovs@guest.arnes.si 